\title{
Combining SERS analysis of serum with PSA levels for improving the detection of prostate cancer
}

\author{
Andrei Stefancu ${ }^{\ddagger 1,2}$, Vlad Moisoiu ${ }^{\ddagger 1,13}$, Razvan Couti ${ }^{4}$, Iulia Andras ${ }^{4,5}$, Razvan Rahota ${ }^{4}$, \\ Dana Crisan ${ }^{6}$, loana E Pavel ${ }^{2,7}$, Carmen Socaciü ${ }^{8,9}$, Nicolae Leopold*,1,2 \& Nicolae Crisan ${ }^{4,5}$ \\ ${ }^{1}$ Faculty of Physics, Babeș-Bolyai University, Cluj-Napoca, Romania \\ ${ }^{2}$ MEDFUTURE Research Center for Advanced Medicine, Iuliu Hatieganu University of Medicine \& Pharmacy, Cluj-Napoca, Romania \\ ${ }^{3}$ IMOGEN Medical Research Institute, County Clinical Emergency Hospital, Cluj-Napoca, Romania \\ ${ }^{4}$ Department of Urology, Clinical Municipal Hospital, Cluj-Napoca, Romania \\ ${ }^{5}$ Department of Urology, luliu Hatieganu University of Medicine \& Pharmacy, Cluj-Napoca, Romania \\ ${ }^{6} 5$ th Medical Clinic, Iuliu Hatieganu University of Medicine \& Pharmacy, Cluj-Napoca, Romania \\ ${ }^{7}$ Department of Chemistry, Wright State University, Dayton, OH 45435, USA \\ ${ }^{8}$ BIODIATECH Research Center for Applied Biotechnology, SC Proplanta, Cluj-Napoca, Romania \\ ${ }^{9}$ Faculty of Food Science \& Technology, University of Agricultural Sciences \& Veterinary Medicine of Cluj-Napoca, \\ Cluj-Napoca, Romania \\ *Author for correspondence: nicolae.leopold@phys.ubbcluj.ro \\ ¥Authors contributed equally
}

Aim: Previous studies regarding surface-enhanced Raman scattering (SERS) of serum have shown promising initial results in discriminating prostate cancer, a strategy which could complement standard tests such as the prostate-specific antigen (PSA). Materials \& methods: SERS spectra of serum samples were combined with serum PSA levels to improve the discrimination accuracy between prostate cancer and nonmalignant pathologies in a cohort of 54 patients using principal component analysis-linear discriminant analysis (PCA-LDA). Results \& discussion: Combining SERS spectra with serum PSA levels in a single PCA-LDA model could discriminate between the two groups with an overall accuracy of $94 \%$, yielding better results than either method alone. Conclusion: These results highlight that combining SERS-based cancer screening with serum PSA levels represents a promising strategy for improving the accuracy of prostate cancer diagnosis.

First draft submitted: 16 April 2018; Accepted for publication: 2 August 2018; Published online: 4 October 2018

Keywords: drop coating deposition • principal component analysis-linear discriminant analysis • prostate cancer • prostate-specific antigen • serum • screening • surface-enhanced Raman scattering

Prostate cancer is the most frequently diagnosed cancer type and the second most common cause of cancer death in male and its prevalence is increasing in developed countries due to the aging of population [1]. The advent of prostate-specific antigen (PSA) testing has improved the diagnosis of this disease, but there is an ongoing debate regarding the opportunity of large scale cancer screening programs. Raman scattering-based assessment of biofluids is emerging as a promising approach for the diagnosis of several types of diseases (including prostate cancer), a strategy which could complement classical screening and diagnostic tests in the future [2].

Screening and early diagnosis are one of the most important means of improving cancer survival, since only limited therapeutic options are available for advanced or metastatic cancer. For example, the 5-year survival rate drops from almost $100 \%$ in the case of localized prostate cancer to about $29 \%$ in the case of cancers that have spread to distant lymph nodes, bones or other organs [3]. In the case of prostate cancer, PSA has been used for decades for cancer screening, risk stratification, response monitoring and relapse detection [4]. In the case of patients which present to the physician with hematuria and other lower urinary tract symptoms, PSA can stratify them in terms of their prostate cancer risk and help deciding the most appropriate action in their management [4]. Elevated PSA levels (above $4 \mathrm{ng} / \mathrm{ml}$ ) and an abnormal digital rectal examination are signs that a malignant prostatic lesion might

Future Medicine 
be present and warrant multiple prostatic biopsies that put the diagnosis. However, PSA levels below the threshold of $4 \mathrm{ng} / \mathrm{ml}$ do not guarantee the absence of disease, the prevalence of prostate cancer in these patients being as high as $15 \%$, of which a significant percentage are high grade tumors [5]. On the other hand, a rise in PSA above threshold can be determined by a multitude of other factors besides prostate cancer, including benign prostatic hyperplasia, prostatitis or urinary tract infections. Given the significant risks of misdiagnosing prostate cancer, the use of PSA for screening in the general population is a much-debated topic and several large prospective trials have tackled this issue. The European Randomized Study of Screening for Prostate Cancer showed some cancer survival benefit in the screened arm [6], whereas the US Prostate, Lung, Colorectal and Ovarian Cancer Screening Trial showed none [7]. A recent paper reanalyzing these trials by accounting for confounding factors and differences in implementation suggested that the contradiction stems from the fact that the standard of care arm, which did not include screening, was heavily contaminated with screened individuals in the Prostate, Lung, Colorectal and Ovarian Cancer Screening trial [8]. The controversy regarding the survival benefit by screening is important due to the consequences of over diagnosing patients [9]. For example, radical prostatectomy and radiotherapy lead to an important increase in the risk of urinary incontinence or erectile and bowel dysfunctions [9].

Given the risk of misdiagnosis by PSA screening, the field could greatly benefit from novel, Raman-based methods of assessing cancer risk, which showed promising initial results, and which could be used in the future for aiding PSA-based diagnostic and screening. Raman spectroscopy is a type of vibrational spectroscopy that uses inelastically scattered monochromatic light for enquiring the molecular structure of samples [10]. The versatility of the method has enabled its use for assessing various biofluids, including serum, urine, tears and cerebrospinal fluid [10]. Previous studies reporting normal Raman spectra of serum samples included pathologies such as prostate [11] and oral cancer [12], among others. One important drawback of Raman spectroscopy is the inherently weak signal of most of the molecules, since only one in $10^{7}$ scattered photons carries information about the molecular structure of the sample.

Acquiring meaningful spectra from biological samples often require additional Raman signal enhancement using techniques such as the drop coating deposition [13], resonant Raman scattering [14], stimulated Raman scattering [15], coherent antistokes Raman scattering [16] or surface-enhanced Raman scattering (SERS) [17,18]. In the case of the drop coating deposition Raman scattering, the spectra are acquired from dried biofluid spots, which contain concentrated compounds. The most soluble compounds will be deposited in the center of the spot, while less soluble substances will be located at the edge. This strategy has been applied in the case of serum [19] and synovial fluid [20], and the spectra were usually dominated by protein-associated bands.

Another widely used amplification method is SERS, which employs metallic substrates such as metallic nanoparticles or other metallic structures with nanoscaled roughness to enhance the Raman signal up to single molecule detection [21-23]. However, enhancing the Raman signal of molecules necessitate their adsorption onto the metallic surface, a chemical process that is particularly complex in the presence of heterogeneous biofluids. The adsorption geometry influences the position and the intensity of the bands, a phenomenon which complicates the interpretation of spectra. SERS-based cancer diagnosis is particularly attractive as a point of care, high-throughput screening strategy, allowing fast decision-making regarding additional diagnostic procedures. Until now, several promising SERS studies of blood (serum) [24], urine [25], saliva [26] or cerebrospinal fluid [27] have been published, of which the majority reported an accuracy above $90 \%$ in discriminating between different groups of interest. In regard to prostate cancer, there have been reports of SERS studies on liquid serum [28,29] and urine [30], as well as one report regarding dried serum [31]. Although these studies demonstrated excellent initial results in terms of accuracy and reproducibility, more investigations are needed to translate this technology in the clinical setting.

The objective of this preliminary study was to assess the prostate cancer diagnosis accuracy yielded by combining SERS and PSA data in the case of patients with elevated PSA levels. To our knowledge, this is the first report of a statistical model combining Raman/SERS spectra with PSA for diagnosis.

\section{Materials \& methods}

This retrospective study has included serum samples acquired during 2014 from 30 prostate cancer patients and 24 controls diagnosed with benign prostatic hyperplasia, prostatitis, atypical small acinar proliferation and prostatic intraepithelial neoplasia based on the pathological results of prostate biopsy cores. All patients presented clinical suspicion for prostate cancer (either PSA $>4 \mathrm{ng} / \mathrm{ml}$ or abnormal digital rectal examination) and underwent prostate biopsy. The serum PSA levels were measured in an accredited laboratory based on electrochemiluminescence (ECLIA). The serum samples were collected before performing any therapy for cancer. Morning blood samples 


\begin{tabular}{|c|c|c|}
\hline & Average age \pm standard deviation & Median PSA (range; ng/ml) \\
\hline Prostate cancer $(n=30)$ & $67 \pm 8$ & $12.1(4.5-121)$ \\
\hline Nonprostate cancer $(n=24)$ & $64 \pm 8$ & $7.4(4.4-21)$ \\
\hline
\end{tabular}

from fasting subjects were collected and then centrifuged at $2000 \mathrm{rot} / \mathrm{min}$ for $10 \mathrm{~min}$ at $18^{\circ} \mathrm{C}$ to isolate serum. The serum was subsequently stored at $-80^{\circ} \mathrm{C}$. The study was approved by the Hospital Ethics Committee and all participants provided written informed consent for enrolling in this study. The research was conducted according to the principles of the Declaration of Helsinki.

\section{Synthesis of SERS-active silver nanoparticles}

Silver nanoparticles were synthesized using the hydroxylamine hydrochloride reduction method [32]. Briefly, $17 \mathrm{mg}$ $\mathrm{H}_{2} \mathrm{NOH} \times \mathrm{HCl}$ and $1.2 \mathrm{ml} \mathrm{NaOH}$ solution (1\%) was added to $8.8 \mathrm{ml}$ ultrapure water. Separately, $17 \mathrm{mg}$ of $\mathrm{AgNO}_{3}$ was dissolved in $90 \mathrm{ml}$ ultrapure water. The $\mathrm{H}_{2} \mathrm{NOH} \times \mathrm{HCl} / \mathrm{NaOH}$ mixture was added rapidly under vigorous stirring to the $\mathrm{AgNO}_{3}$ solution to synthesize the nanoparticles. The $\mathrm{pH}$ of the silver colloid was measured before the SERS measurements using a conventional $\mathrm{pH}$ strip and was found to be 7.5 . The fresh colloid was left overnight at room temperature before measurements. All chemicals were purchased from Sigma-Aldrich. The silver nanoparticles were characterized by UV-Vis spectroscopy (Jasco V-630 Spectrometer, $2 \mathrm{~nm}$ spectral resolution, Pfungstadt, Germany) and transmission electronic microscopy (TEM) (JEOL, JEM-100CX, operating at $100 \mathrm{kV}$, Tokyo, Japan). Before the measurements, $2 \mathrm{ml}$ of silver nanoparticles were concentrated by centrifuging them for $15 \mathrm{~min}$ at $5800 \mathrm{~g}$ and then resuspended in $50 \mu \mathrm{l}$ ultrapure water. Then, $1 \mu \mathrm{l}$ of serum was mixed gently with $9 \mu \mathrm{l}$ of concentrated colloid and deposited onto microscope slides covered with aluminum foil and left to dry for $20 \mathrm{~min}$. The SERS spectra were acquired from the rim of the spot at room temperature.

SERS spectra were analyzed in the $606-1715 \mathrm{~cm}^{-1}$ range using an InVia Reflex Spectrometer (Renishaw, Gloucestershire, UK) based on an upright Leica microscope equipped with a spectrograph with 1200 lines $/ \mathrm{mm}$ grating. A diode laser emitting at $785 \mathrm{~nm}(\sim 16 \mathrm{~mW}$ on the sample) was focused on the rim of the dried samples using a $5 \times$ objective (Olympus, NA = 0.12) for $40 \mathrm{~s}$. The calibration was based on the $520 \mathrm{~cm}^{-1}$ Raman line of an internal Si standard. The samples were chosen randomly and for each sample two measurements were averaged.

\section{Data analysis}

Data analysis was performed using The Unscrambler (X version 10.1, CAMO Software, Oslo, Norway) and it consisted of background removal by linear baseline correction, followed by mean normalization and principal component analysis-linear discriminant analysis (PCA-LDA) using the first 15 principal components (PCs). PCA reduces the dimensionality of the data by projecting all spectra onto PCs. Linear discriminant analysis is a supervised statistical analysis method that requires prior knowledge regarding the type of sample (e.g., cancer or control) for building a mathematical model that can discriminate between the sample type. To combine spectral information with PSA levels, the PSA values were normalized by dividing them to their median value. Next, the normalized values were appended to the SERS spectra matrix and the PCA-LDA was performed on the resulting matrix of data. The D’Agostino \& Pearson omnibus normality tests, unpaired t-test, Mann-Whitney nonparametric tests and receiver operating characteristic (ROC) curve analysis were performed with GraphPad Prism (Version 6.0, GraphPad Software).

\section{Results}

In this study, we included $n=30$ samples from patients diagnosed with prostate cancer and $n=24$ samples from patients with a diversity of nonmalignant diseases that were biopsied due to suspicion of prostate cancer but for which the pathology results demonstrated the absence of prostate cancer. The control group included five patients with benign prostatic hyperplasia, five patients with prostatitis, one patient with atypical small acinar proliferation, one patient with intraepithelial neoplasia and 12 patients with various combinations of the above mentioned disorders (Supplementary Table 1).

Data regarding the age and the serum PSA levels of the patients enrolled in our study is presented in Table 1 . The age of the patients had a normal distribution (D'Agostino \& Pearson omnibus normality test) and the difference 


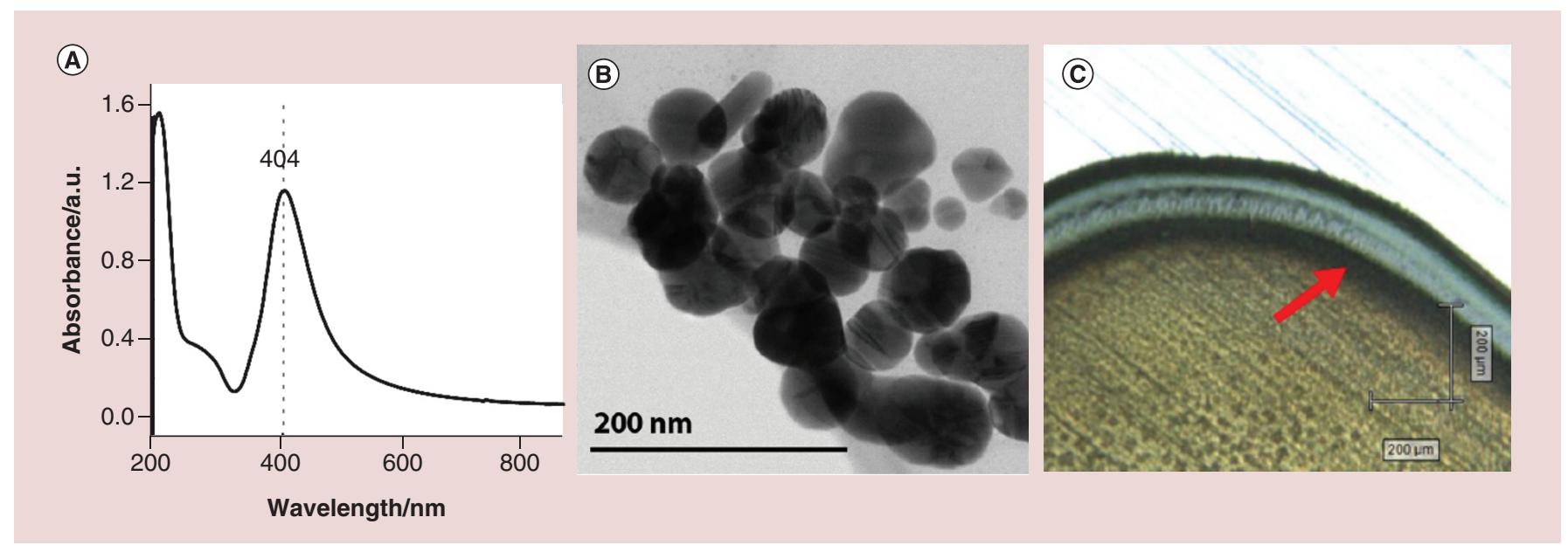

Figure 1. The physical properties of the setup used for acquiring surface-enhanced Raman scattering spectra. The UV-Vis spectrum depicting the resonance plasmonic band at $404 \mathrm{~nm}(\mathrm{~A})$, a characteristic transmission electronic microscopy micrograph of the silver nanoparticles (B), a typical spot created by a dried sample (C). The surface-enhanced Raman scattering spectra were acquired from the outer rim of the spot (see red arrow).

\begin{tabular}{|c|c|c|}
\hline \multirow[b]{2}{*}{ Predicted } & \multicolumn{2}{|c|}{ Reference } \\
\hline & Prostate cancer & Controls \\
\hline Prostate cancer & 29 & 4 \\
\hline Controls & 1 & 20 \\
\hline
\end{tabular}

between the two groups was not statistically significant (unpaired t-test assuming equal variances; $\mathrm{p}>0.05$ ). The PSA values followed a nonparametric distribution (D'Agostino \& Pearson omnibus normality test; $\mathrm{p}<0.001$ for both groups) and there was a statistically significant difference between the prostate cancer and control group (Mann-Whitney nonparametric test; $\mathrm{p}<0.001$ ). Other information regarding patients such as histological type, Gleason score or extraprostatic extension can be found in the Supplementary Table 1.

The UV-Vis spectrum of the colloidal solution used for SERS measurements (Figure 1A) showed a plasmonic band at $404 \mathrm{~nm}$. Figure $1 \mathrm{~B}$ shows a representative TEM micrograph of the nanoparticles.

The SERS spectra were acquired from dried spots of serum and nanoparticle mixtures, thus combining the SERS method with the drop coating deposition method. Figure $1 \mathrm{C}$ shows a part of a sample spot resulting due to the 'coffee ring' effect. The SERS signal of serum metabolites was acquired from the periphery of the spot (Figure 1C, red arrow).

Figure 2 shows the effect of processing by baseline subtraction and normalization on SERS spectra from prostate cancer (Figure 2A \& C) and control samples (Figure 2B \& D).

The mean SERS spectra from patients diagnosed with prostate cancer as well as controls, along with their difference is depicted in Figure 3. Most of the SERS bands of serum could be tentatively attributed to purine metabolites such as uric acid and hypoxanthine $\left(639,727,810,887,1135,1203,1262,1566 \mathrm{~cm}^{-1}\right)$ [33], while other bands, such as the ones from 1002 to $1650 \mathrm{~cm}^{-1}$ could be identified as the phenylalanine breathing mode and the Amide I band, respectively.

Next, we used PCA-LDA to quantitatively assess the SERS-based discrimination accuracy. The results of the prediction matrix along with the line that separates the samples attributed to the cancer group from control samples are depicted in Figure 4. The confusion matrix corresponding to the prediction matrix is presented in Table 2.

The specificity of the PCA-LDA model, which is defined as the number of true positive prostate cancer samples from the total number of cancer samples, was $96 \%$, while sensitivity, which is defined as the number of true negative cancer samples from the total number of cancer-negative samples, was $83 \%$. The overall accuracy of the model, which is defined as the percentage of correctly assigned samples, was approximately $90 \%$. 

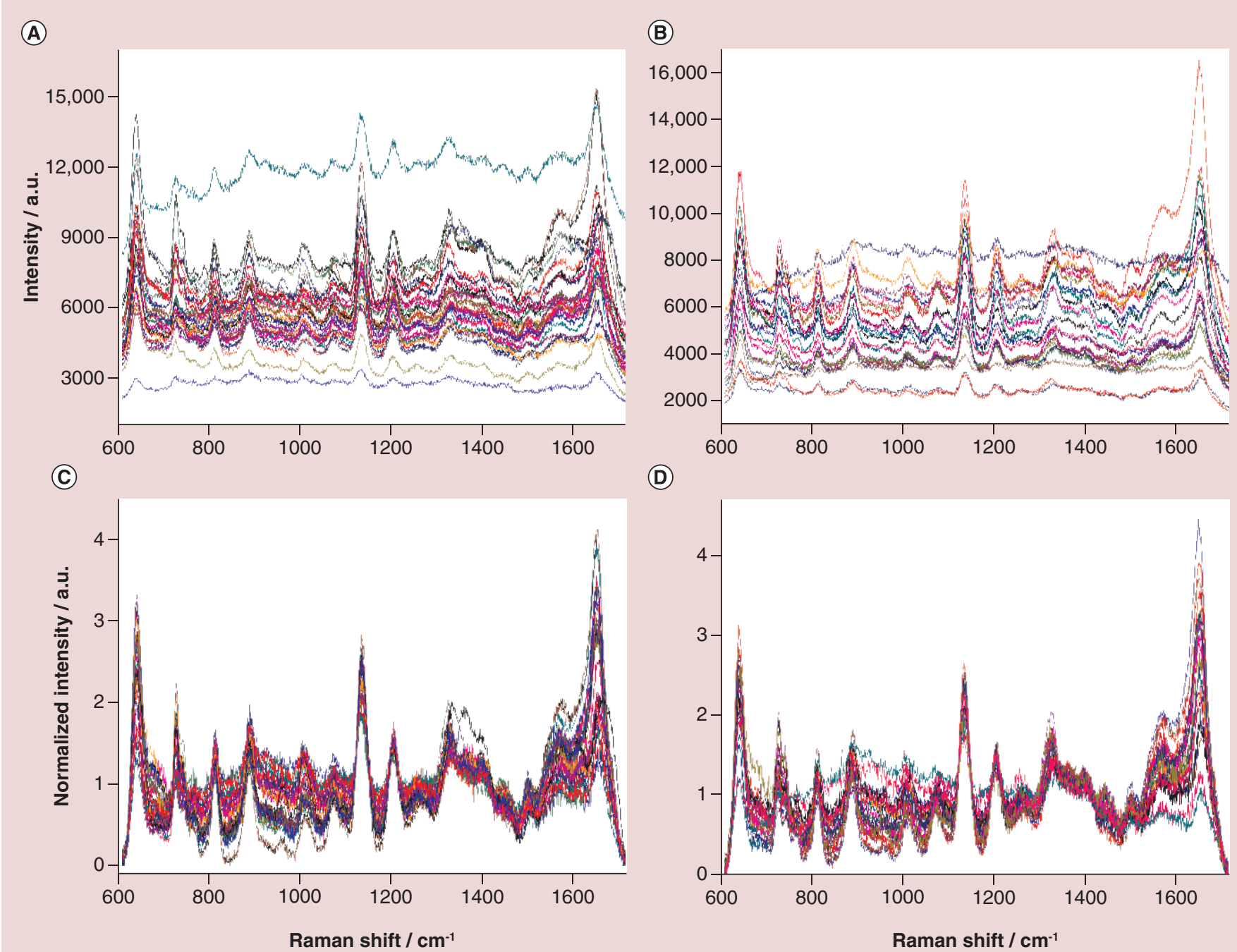

(D)

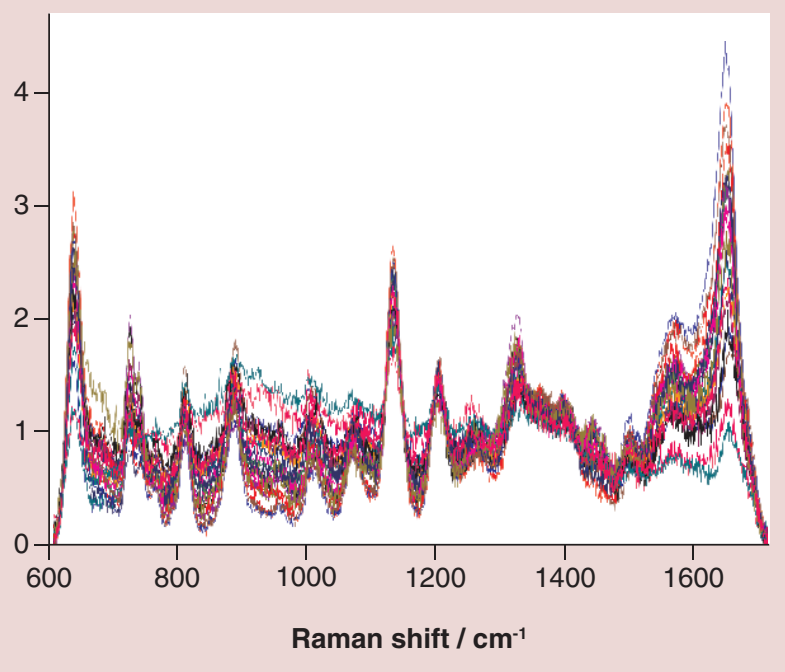

Figure 2. The effect of normalization on surface-enhanced Raman spectroscopy spectra. The raw surface-enhanced Raman scattering spectra of prostate cancer $(n=30)(A)$ and control samples $(n=24)(B)$, surface-enhanced Raman scattering spectra of prostate cancer (C) and control samples (D) after background removal and mean normalization.

Figure 3. Mean surface-enhanced Raman scattering spectra of controls (ctrl, $\mathrm{n}=23$ ) and prostate cancer samples (pc, $n=31$ ) and their difference (dif), as indicated in the figure.

CTRL: Control; PC: Prostate cancer.

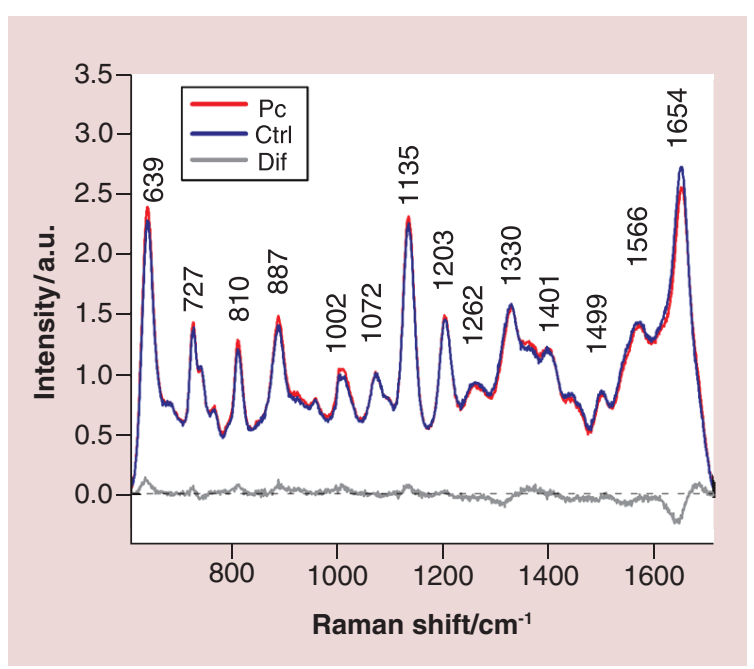




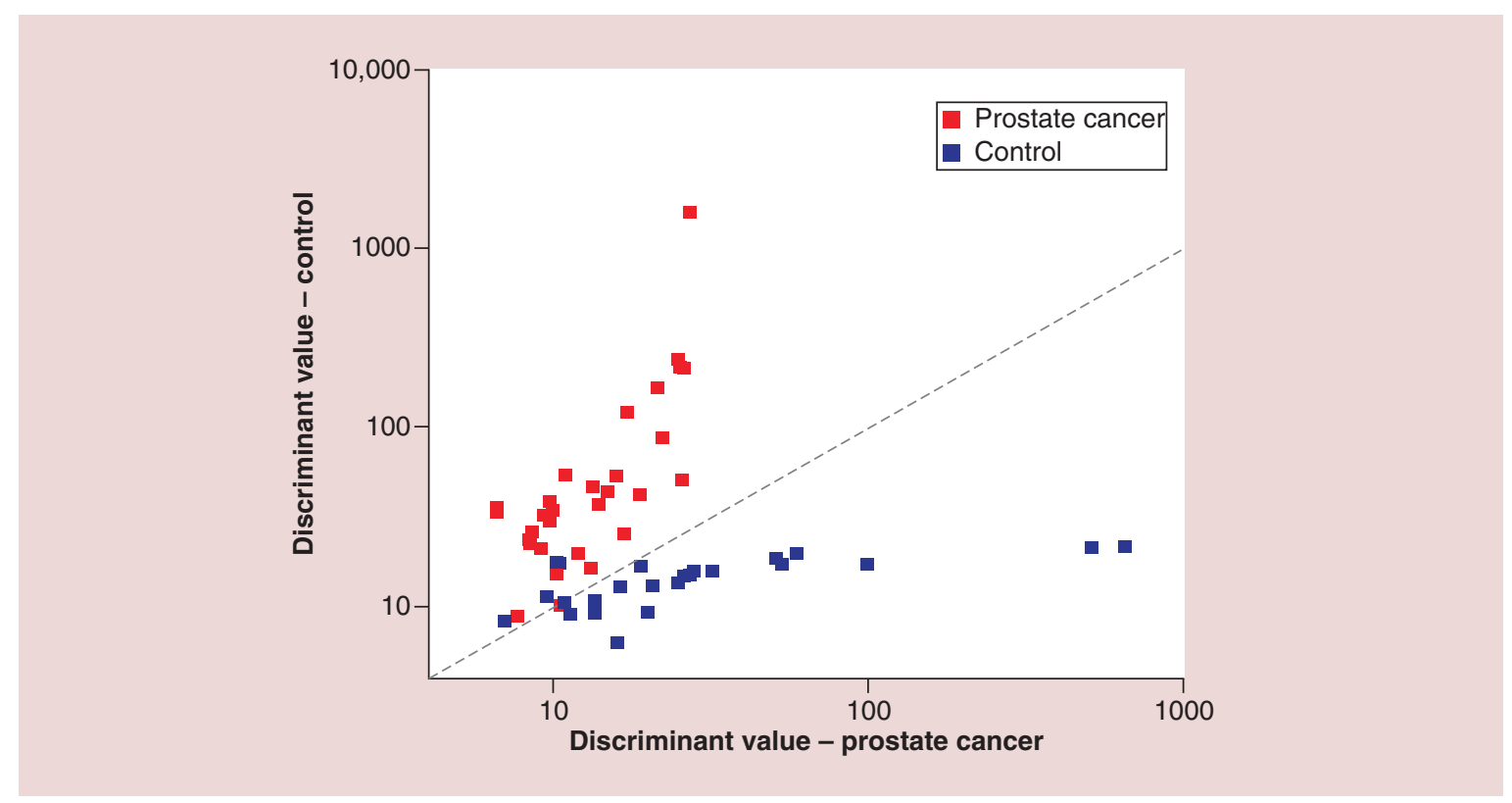

Figure 4. The discriminant values of the principal component analysis-linear discriminant analysis model involving surface-enhanced Raman scattering spectra. The decision line separating points corresponding to prostate cancer samples $(n=30)$ from points corresponding to control samples $(n=24)$. The values presented in the plot are the negatives of the initial values, a transformation needed for the logarithmic representation.

CTRL: Control; PC: Prostate cancer.

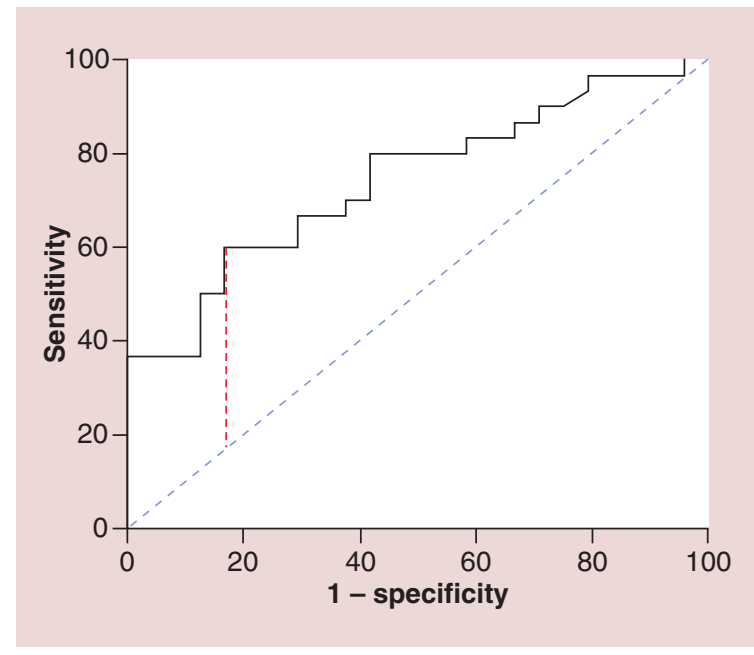

Figure 5. The receiver operating characteristic curve obtained for the prostate-specific antigen values. The area under the curve is 0.77 , while the sensitivity and specificity corresponding to a cut-off value of $11 \mathrm{ng} / \mathrm{ml}$ are 61 and $87 \%$, respectively. The chosen cut-off value corresponds to the point on the curve that is farthest away from the diagonal line in such a way that the sensitivity and specificity are simultaneously maximized.

The receiver operating characteristic (ROC) curve obtained with the PSA values of the patients enrolled in our study is presented in Figure 5.

The area under the curve of the ROC was 0.74 , and by setting a PSA cut-off value of $11 \mathrm{ng} / \mathrm{ml}$, the sensitivity and specificity was $60 \%$ and $83.3 \%$, respectively, while the overall accuracy was $70.4 \%$. The chosen cut-off value corresponds to the point on the ROC curve, which is farthest away from the diagonal line, such that the sensitivity and specificity are simultaneously maximized. The confusion matrix obtained by setting a cut-off value of $11 \mathrm{ng} / \mathrm{ml}$ is shown in Table 3.

Next, we sought to combine the spectral data with the information gained from PSA in a single model. To do so, the PSA values were first normalized by dividing them with their median and then appended at the end of the SERS spectra. The resulting string of information, containing both PSA values and spectral information, was then used to discriminate between the two groups using PCA-LDA. 
Table 3. Confusion matrix obtained by setting a cut-of value of $11 \mathrm{ng} / \mathrm{ml}$ for prostate-specific antigen level.

\begin{tabular}{llc} 
& & Reference \\
Predicted & Prostate Cancer & Controls \\
Prostate cancer & 18 & 4 \\
\hline Controls & 12 & 20 \\
\hline
\end{tabular}

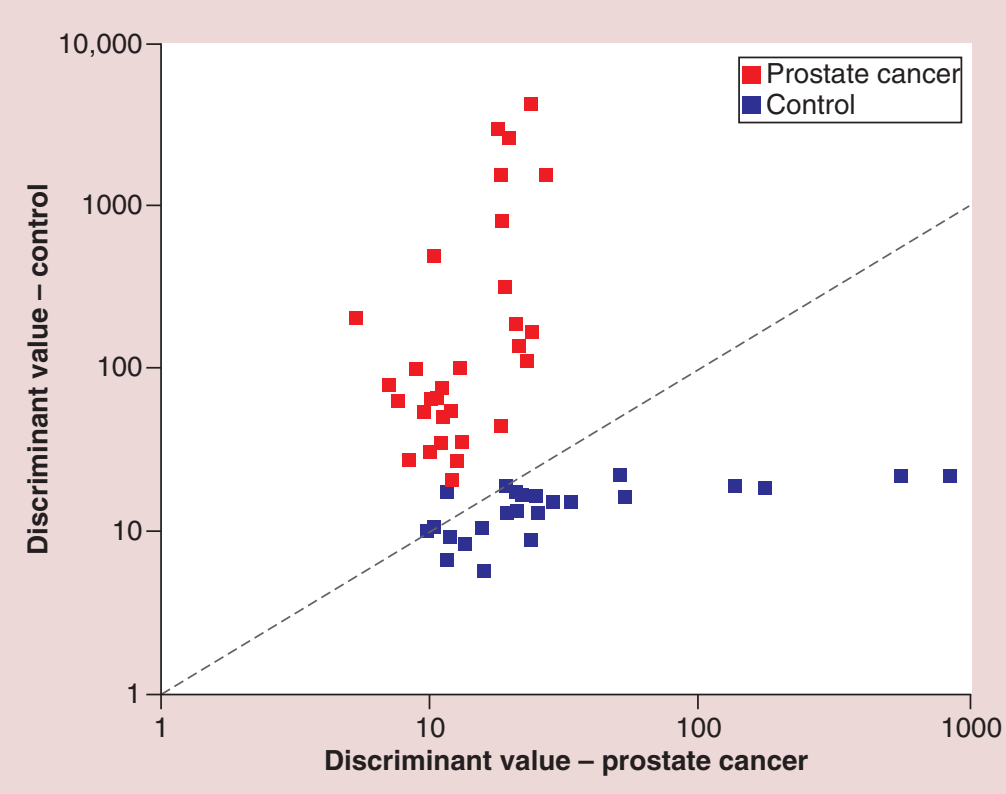

Figure 6. The discriminant values of the principal component analysis-linear discriminant analysis model involving surface-enhanced Raman scattering spectra and prostate-specific antigen values. The decision line separating points corresponding to prostate cancer samples $(n=30)$ from points corresponding to control samples $(n=24)$. The values presented in the plot are the negatives of the initial values, a transformation needed for the logarithmic representation.

Table 4. Confusion matrix obtained from the principal component analysis-linear discriminant analysis model involving serum surface-enhanced Raman scattering spectra and prostate- specific antigen values.

\begin{tabular}{lll} 
& \multicolumn{2}{c}{ Reference } \\
\cline { 2 - 3 } Predicted & Prostate cancer & Controls \\
\hline Prostate cancer & 30 & 3 \\
\hline Controls & 0 & 21 \\
\hline
\end{tabular}

The resulting discriminant matrix of the PCA-LDA performed on the mixed string of information is presented in Figure 6, while the corresponding confusion matrix is presented in Table 4.

The sensitivity and specificity yielded by the PCA-LDA on the mixed string of information was 87 and $100 \%$, respectively, while the overall accuracy was $94 \%$.

\section{Discussion}

In clinical practice, the challenge is to discriminate between prostate cancer and other pathologies that can raise PSA levels, such as benign prostatic hyperplasia, prostatitis, atypical small acinar proliferation and prostatic intraepithelial neoplasia. In order to mimic the real clinical setting, instead of healthy volunteers, we considered a control group consisting of 24 patients with diverse nonmalignant diseases that were biopsied due to suspicion of prostate cancer but for which the pathology results demonstrated the absence of prostate cancer. Some previous reports regarding SERS of serum or urine for prostate cancer detection have used healthy volunteers as controls [11,34], whereas other reports mimicked the clinical setting by enrolling patients with benign prostatic hyperplasia as controls $[28,29]$. The 
control group is particularly important in the case of Raman/SERS studies of biofluids, since the subtle differences in metabolites are rather nonspecific, and there is always a risk that the spectral pattern is shared by multiple diseases. For example, spectral features involving high purine metabolites could be shared by diseases ranging from cancer [35], hyperthyroidism [36] or gout [37], and therefore, it is of uttermost importance to know that SERS performs well in a setting that mimics the clinical setting.

Silver colloidal nanoparticles obtained by hydroxylamine reduction were preferred in several studies concerning SERS investigation of biofluids [38-40]. The silver nanoparticles are already activated by chloride ions present in the colloidal solution after the synthesis reaction, leading to intense SERS spectra of the adsorbed molecules. The SERS spectra were acquired using the drop coating deposition method. During the drying process, a capillary outer flow concentrates all metabolites at the edge of the drop, which are then deposited in concentric peripheric rings.

A previous study comparing the SERS spectra of serum acquired using several types of metallic colloids showed that using hydroxylamine hydrochloride reduced silver nanoparticles leads to reproducible spectra irrespective of the excitation wavelength [41]. The same study reported that among various excitation wavelengths, the $785 \mathrm{~nm}$ laser line ensures the best repeatability. However, the use of lower wavelengths, such as the 532 or $514 \mathrm{~nm}$ laser, can be advantageous in certain situations, due to the enhancement of carotenoid associated bands. In our case, preliminary experiments showed that the best signal-to-noise ratios are obtained with the $785 \mathrm{~nm}$ laser line, also (data not shown).

The mean SERS spectra from patients diagnosed with prostate cancer as well as controls, along with their difference is depicted in Figure 3. The SERS bands of serum could be tentatively attributed to purine metabolites such as uric acid and hypoxanthine [33], while other bands, such as the ones from 1002 and $1650 \mathrm{~cm}^{-1}$ could be identified as the phenylalanine breathing mode the Amide I band, respectively. Previous studies have attributed similar bands to metabolites ranging from carbohydrates such as D-Mannose to fibrillar proteins such as collagen [42]. The assignment of bands is a critical issue of SERS studies on biofluids, due to the complexity of the chemical composition and the fact that the position and intensity of bands varies with the laser wavelength, nanoparticles type and other experimental conditions [41]. Compared with previous SERS studies on liquid samples, the position and intensity of the bands reported by us are almost identical $[28,29]$, although slight differences in wavenumbers and relative intensity exist stemming from variations in the experimental settings. The similarity could be explained by the fact that the SERS spectra are preponderantly due to small metabolite molecules, for which conformational changes are less important than for proteins. However, compared with a SERS study on dried serum samples [31], there are many bands that do not have clear correspondence, except for some few common bands at 721 and $1135 \mathrm{~cm}^{-1}$. In the case of the spontaneous Raman study on liquid serum samples [11], the only distinct bands in the spectra were attributed to carotenoids that were differentially expressed between the prostate cancer serum samples and healthy controls (intense bands around 1155 and $1523 \mathrm{~cm}^{-1}$ ).

In order to assess the classification accuracy yielded by the SERS spectra, we employed PCA-LDA, which was the method of choice in the majority of previous SERS and Raman studies on biofluids (see below). The reduction of the sample dimensionality by PCA is needed, since LDA can only be applied if the number of samples is larger than the number of variables (in our case, 1011 wavenumbers in the $606-1715 \mathrm{~cm}^{-1}$ range). Therefore, by coupling PCA to LDA, the later does not consider the actual spectra of each sample but only the scores values resulting from PCA. For each sample, LDA computes the class discriminant value and then attributes the sample to the class having the highest value. Note that the discriminant values can also be negative.

In the case of the PCA-LDA model obtained from SERS spectra, the specificity was $96 \%$, while the sensitivity was $83 \%$. The overall accuracy of the model, defined as the percentage of correctly assigned samples, was approximately $90 \%$. These results confirm previous studies and demonstrate that multivariate data analysis is efficient in discriminating between prostate cancer samples and controls. For example, in a large study involving 120 participants, prostate cancer and a control group with PSA levels between 4 and $10 \mathrm{ng} / \mathrm{ml}$ (the so called 'gray zone') were classified using PCA-LDA with an overall accuracy of 94\% [28]. This study compared the spectra from prostate cancer patients with both healthy volunteers and patients diagnosed with benign prostatic hyperplasia, a cohort that better simulates the clinical setting. Another recent study reported a high throughput normal Raman system that correctly diagnosed prostate cancer with $96 \%$ accuracy on a smaller cohort $(\mathrm{n}=20$ patients) using PCA-LDA, but in this case, the controls were represented by healthy volunteers [11]. A SERS study performed on both serum and expressed prostatic secretion (separately) reported an accuracy of $68 \%$ for the serum and $75 \%$ for expressed prostatic secretion using PCA-LDA ( $\mathrm{n}=40$ patients) [43], while SERS spectra acquired from urine samples lead an overall accuracy of $95 \%$ in a small cohort using PCA-LDA $(n=18)$ [30]. One SERS study on dried serum samples 
reported an overall accuracy of $98 \%$ in discriminating 93 prostate cancer patients from 68 healthy volunteers using support vector machines, which is another widely used multivariate statistical analysis method [32]. These results suggest that Raman spectroscopy is a promising method of diagnosing prostate cancer that could enable accurate high-throughput screening in the future. However, validating Raman or SERS-based strategies will require prospective studies performed in the clinical setting on large groups, and for the moment, no such study has been undertaken. Moreover, accomplishing such a task in a multicentric trial will require homogenized experimental protocols, which can represent an important hurdle given the sensibility of SERS to even slight differences in the experimental setting.

PSA-based screening, which is the gold standard for screening in prostate cancer, is known to yield a considerable number of false positive results, which prompt additional testing for ruling out prostate cancer, including invasive procedures such as biopsy. Moreover, some correctly diagnosed prostate cancers are indolent and slowly growing, meaning that the harm of diagnosing and treating these cases may outweigh any likely benefit [9]. The ROC curve obtained with the PSA values of the patients enrolled in our study is presented in Figure 5, and it corresponds to a specificity of $83.3 \%$, a sensitivity of $60 \%$ and an overall accuracy of $70.4 \%$. These values are comparable with the ones reported in a longitudinal cohort showing that setting cut-off values of 3,4 and $5 \mathrm{ng} / \mathrm{ml}$ leads to an estimated sensitivity of 59, 44 and 33\% respectively, while the specificity estimates were 87, 92 and 95\%, respectively [44]. The authors concluded that no single cut-off values attained an accuracy formally required for a screening test and therefore, finding means of improving diagnostic accuracy of PSA are needed. It should be mentioned that all patients enrolled in our study were biopsied based on abnormal PSA levels (PSA $>4 \mathrm{ng} / \mathrm{ml}$ ), and therefore, the PSA values of all the patients were above $4 \mathrm{ng} / \mathrm{ml}$ by default.

Next, we sought to combine the spectral data with the information gained from PSA in a single model. To do so, the PSA values were first normalized by dividing them with their median and then appended at the end of the SERS spectra. The normalization was needed since the absolute PSA values were too high compared with the intensity values of the SERS spectra, leading to the spectral information being neglected by the mathematical model. The resulting string of information, containing both PSA values and spectral information, was then used to discriminate between the two groups using PCA-LDA.

The resulting confusion matrix (Table 4) corresponds to a specificity of $100 \%$, a sensitivity of $87 \%$ and an overall accuracy of $94 \%$. Compared with the $90 \%$ overall accuracy obtained in the PCA-LDA model involving only spectral information and to the $70.4 \%$ accuracy obtained by setting a PSA cut-off value of $11 \mathrm{ng} / \mathrm{ml}$, the combined model provided complementary information, leading to better results than either of the methods alone.

The strategy of combining classical markers with spectral data has been insufficiently explored and to our knowledge, no such study has been reported so far. Therefore, our study opens new avenues in cancer screening, by showing that spectral data can be successfully combined with the information provided by classical tumor markers to yield better results. Combinations of single or multiple tumor markers with stage, grade, demographic, epidemiologic, genetic or clinical data are increasingly used for improving the diagnostic or prognostic accuracy. For example, the risk of ovarian malignancy algorithm that incorporates CA125, HE4 and menopausal status has been approved by the US FDA in the case of patients presenting with a pelvic mass [45], while a panel of six tumor markers (CEA, [CA15-3], [SCCA], [CYFRA 21-1], neuron-specific enolase and ProGRP) was shown to be more accurate in predicting lung cancer than any single tumor marker alone [46]. In the case of prostate cancer, combining PSA levels with the percentage of free PSA was shown to reduce unnecessary biopsies by $20 \%$ in patients with no palpable prostate nodule and PSA levels in the gray zone [47]. Similarly, a recent prospective population-based diagnostic study (The Stockholm 3 [STHLM3]) combining plasma protein biomarkers (including PSA and free PSA), genetic polymorphisms and clinical variables such as age, family, history, previous prostate biopsy, prostate exam and the number of biopsies showed that using all these variables could reduce the number of benign biopsies by $32 \%$ (95\% CI: 24-39\%) [48,49]. Several other models are also used in both malignant and nonmalignant conditions [50], proving that combining heterogeneous markers is a sound strategy for increasing the accuracy.

Our study suggests that in the future, prostate cancer associated spectral features could complement other markers and disease parameters, another step toward personalized risk-based prostate cancer management. Given the high number of tumor markers used for other types of cancer, our work could also inspire future studies that aim to combine spectral data with validated tumor markers for improving the accuracy of cancer diagnosis. 


\section{Conclusion}

Prostate cancer is one of the leading causes of death in developed countries, for which screening is of questionable benefit using current methods such as PSA, despite several large clinical trials that tackled the issue. In this study, we showed that combining PSA levels with SERS spectra acquired from serum samples yielded better results (overall accuracy 94\%) than either of the methods alone in discriminating between patients with prostate cancer and controls with elevated serum PSA levels. Our study represents another step toward personalized risk-based management of prostate cancer, which could inspire subsequent research assessing the combination of spectral features with other classical tumor markers for improving the accuracy of cancer detection.

\section{Future perspective}

The number of reports regarding Raman and SERS studies of biofluids has increased considerably in the last years, highlighting the immense potential of this method as a high-throughput tool for detecting cancer and other diseases. The studies undertaken so far have focused on a single disease or pathology, but the heterogeneity of the methods used for acquiring the spectra prevents a direct comparison between the data. Therefore, there is a need for studies comparing multiple pathologies in respect to their characteristic spectrum and a better collaboration between the scientists in homogenizing the experimental settings. Proving the diagnostic capabilities of Raman/SERS-based strategies will also require prospective enrolling of patients in large multicentric clinical trials. When these issues will be surpassed, it is possible that disease-associated spectral features could be integrated in risk scores and models, which for the moment, use only tumor marker levels and clinical parameters.

\section{Summary points}

Background

- Prostate-specific antigen (PSA) is a serum tumor marker that is widely used for the early detection of prostate cancer, but its low accuracy has raised controversies regarding its suitability for screening in the general population.

- Surface-enhanced Raman scattering (SERS) is emerging as a novel method for assessing the differences in chemical composition of serum that has demonstrated promising initial results.

\section{Experimental}

- Serum samples from 30 prostate cancer patients and 24 controls diagnosed with nonmalignant diseases were collected and mixed with silver nanoparticles and then left to dry on aluminum substrates.

- SERS spectra were acquired from the rim of the dried spots.

- The accuracy of principal component analysis-linear discriminant analysis (PCA-LDA) in discriminating between prostate cancer and controls was assessed using either spectral data alone or in combination with PSA.

Results \& discussion

- In terms of the SERS spectra, a comparison between the prostate cancer and control groups showed differences in bands associated with purine derivatives and proteins, which can be employed to discriminate between the two groups.

- The PCA-LDA model that combined both spectral features and PSA yielded superior results (overall accuracy $94 \%$ ) compared with the PCA-LDA model that considered only spectra features (overall accuracy $90 \%$ ) and to PSA (overall accuracy $70.4 \%$ using a cut-off value of $11 \mathrm{ng} / \mathrm{ml}$ ).

- Combining SERS/Raman spectral features with classical tumor markers such as PSA represents a novel strategy for increasing diagnostic accuracy of prostate cancer.

- This study could inspire future studies involving Raman/SERS spectra of biofluids and other approved tumor markers.

Conclusion

- Multivariate data analysis of serum SERS spectra and PSA values from prostate cancer and control patients yielded better results than either of the methods alone.

- SERS-based cancer screening on serum samples could complement in the future classical tumor markers such as PSA. 
Authors' contributions

N Crisan, N Leopold, C Socaciu and IE Pavel designed and supervised the study; R Couti, I Andras, R Rahota, D Crisan and N Crisan enrolled patients and provided patient samples. A Stefancu and $V$ Moisoiu performed the Raman measurements and the biostatistical analyses. A Stefancu and $V$ Moisoiu wrote the manuscript, with input from all authors. All authors have revised the final version.

Acknowledgments

A Stefancu and M Vlad acknowledge support from the College for Advanced Performance Studies, Babeș-Bolyai University, ClujNapoca, Romania. The authors would like to acknowledge Z Bálint for his valuable input regarding study design, data analysis and manuscript writing.

Financial \& competing interests disclosure

Financial support from the Competitiveness Operational Programme 2014-2020 (POC-A1-A1.1.4-E-2015), financed under the European Regional Development Fund, project number P_37_765 is highly acknowledged. The authors have no other financial involvement with any organization or entity with a financial interest in or financial conflict with the subject matter or materials discussed in the manuscript apart from those disclosed.

No writing assistance was utilized in the production of this manuscript.

Ethical conduct of research

The authors state that they have obtained ethical approval from the Clinical Municipal Hospital Cluj-Napoca Ethics Committee and that the research followed the principles outlined in the Declaration of Helsinki for all human or animal experimental investigations. All participants provided written informed consent for enrolling in this study.

Open access

This work is licensed under the Attribution-NonCommercial-NoDerivatives 4.0 Unported License. To view a copy of this license, visit http://creativecommons.org/licenses/by-nc-nd/4.0/

\section{References}

1. Siegel RL, Miller KD, Jemal A. Cancer statistics, 2016. CA Cancer J. Clin. 66(1), 7-30 (2016).

2. Bonifacio A, Cervo S, Sergo V. Label-free surface-enhanced Raman spectroscopy of biofluids: fundamental aspects and diagnostic applications. Anal. Bioanal. Chem. 407(27), 8265-8277 (2015).

3. Harbord M, Eliakim R, Bettenworth D et al. Third European evidence-based consensus on diagnosis and management of ulcerative colitis. part 2: current management. J. Crohns Colitis 11(7), 769-784 (2017).

4. Parker C, Gillessen S, Heidenreich A, Horwich A. Cancer of the prostate: ESMO Clinical Practice Guidelines for diagnosis, treatment and follow-up. Ann. Oncol. 26, (Suppl 5), v69-v77 (2015).

5. Thompson IM, Pauler DK, Goodman PJ et al. Prevalence of prostate cancer among men with a prostate-specific antigen level < or = $4.0 \mathrm{ng}$ per milliliter. N. Engl. J. Med. 350(22), 2239-2246 (2004).

6. Schröder FH, Hugosson J, Roobol MJ et al. Prostate-cancer mortality at 11 years of follow-up. N. Engl. J. Med. 366(11), 981-990 (2012).

7. Andriole GL, Crawford ED, Grubb RL, 3rd et al. Prostate cancer screening in the randomized prostate, lung, colorectal, and ovarian cancer screening trial: mortality results after 13 years of follow-up. J. Natl Cancer Inst. 104(2), 125-132 (2012).

8. Tsodikov A, Gulati R, Heijnsdijk EaM et al. Reconciling the effects of screening on prostate cancer mortality in the ERSPC and PLCO Trials. Ann. Intern. Med. 167(7), 449-455 (2017).

9. Wilt TJ, Jones KM, Barry MJ et al. Follow-up of prostatectomy versus observation for early prostate cancer. N. Engl. J. Med. 377(2), 132-142 (2017).

10. Butler HJ, Ashton L, Bird B et al. Using Raman spectroscopy to characterize biological materials. Nat. Protoc. 11, 664 (2016).

11. Medipally DK, Maguire A, Bryant J et al. Development of a high throughput (HT) Raman spectroscopy method for rapid screening of liquid blood plasma from prostate cancer patients. Analyst 142(8), 1216-1226 (2017).

12. Sahu A, Sawant S, Mamgain H, Krishna CM. Raman spectroscopy of serum: an exploratory study for detection of oral cancers. Analyst 138(14), 4161-4174 (2013).

13. Pazderka T, Kopecký V. Drop coating deposition Raman spectroscopy of proteinogenic amino acids compared with their solution and crystalline state. Spectrochim. Acta A Mol. Biomol. Spectrosc. 185, 207-216 (2017).

14. Efremov EV, Ariese F, Gooijer C. Achievements in resonance Raman spectroscopy review of a technique with a distinct analytical chemistry potential. Anal. Chim. Acta 606(2), 119-134 (2008). 
Research Article Stefancu, Moisoiu, Couti et al.

15. Prince RC, Frontiera RR, Potma EO. Stimulated Raman Scattering: From bulk to nano. Chem. Rev. doi:10.1021/acs.chemrev.6b00545 (2016). (Epub ahead of print).

16. Moura CC, Tare RS, Oreffo ROC, Mahajan S. Raman spectroscopy and coherent anti-stokes Raman scattering imaging: prospective tools for monitoring skeletal cells and skeletal regeneration. J. R. Soc. Interface 13(118), 20160182 (2016).

17. Mosier-Boss PA. Review of SERS substrates for chemical sensing. Nanomaterials 7(6), 142 (2017).

18. Jun B-H, Kim G, Noh MS et al. Surface-enhanced Raman scattering-active nanostructures and strategies for bioassays. Nanomedicine 6(8), 1463-1480 (2011).

19. Neugebauer U, Trenkmann S, Bocklitz T, Schmerler D, Kiehntopf M, Popp J. Fast differentiation of SIRS and sepsis from blood plasma of ICU patients using Raman spectroscopy. J. Biophotonics 7(3-4), 232-240 (2014).

20. Esmonde-White KA, Mandair GS, Raaii F et al. Raman spectroscopy of synovial fluid as a tool for diagnosing osteoarthritis. J. Biomed. Opt. 14(3), 034013 (2009).

21. Sharma B, Frontiera RR, Henry A-I, Ringe E, Van Duyne RP. SERS: materials, applications, and the future. Mater. Today 15(1), 16-25 (2012).

22. Kircher MF. How can we apply the use of surface-enhanced Raman scattering nanoparticles in tumor imaging? Nanomedicine 12(3), 171-174 (2017).

23. Sha MY, Xu H, Penn SG, Cromer R. SERS nanoparticles: a new optical detection modality for cancer diagnosis. Nanomedicine 2(5), 725-734 (2007).

24. Vargas-Obieta E, Martinez-Espinosa JC, Martinez-Zerega BE, Jave-Suarez LF, Aguilar-Lemarroy A, Gonzalez-Solis JL. Breast cancer detection based on serum sample surface enhanced Raman spectroscopy. Lasers Med. Sci. 31(7), 1317-1324 (2016).

25. Bhattacharjee T, Khan A, Maru G, Ingle A, Krishna CM. A preliminary Raman spectroscopic study of urine: diagnosis of breast cancer in animal models. Analyst 140(2), 456-466 (2015).

26. Li X, Yang T, Lib S, Yu TEDRN, Popp J. Surface-enhanced Raman spectroscopy differences of saliva between lung cancer patients and normal people. Presented at: Clinical and Biomedical Spectroscopy and Imaging II. Munich, Germany (14 June 2011).

27. Sathyavathi R, Dingari NC, Barman I et al. Raman spectroscopy provides a powerful, rapid diagnostic tool for the detection of tuberculous meningitis in ex vivo cerebrospinal fluid samples. J. Biophotonics 6(8), 567-572 (2013).

28. Chen N, Rong M, Shao X et al. Surface-enhanced Raman spectroscopy of serum accurately detects prostate cancer in patients with prostate-specific antigen levels of 4-10 ng/mL. Int. J. Nanomedicine 12, 5399-5407 (2017).

29. Shao X, Pan J, Wang Y et al. Evaluation of expressed prostatic secretion and serum using surface-enhanced Raman spectroscopy for the noninvasive detection of prostate cancer, a preliminary study. Nanomed. Nanotechnol. Biol. Med. 13(3), 1051-1059 (2017).

30. Del Mistro G, Cervo S, Mansutti E et al. Surface-enhanced Raman spectroscopy of urine for prostate cancer detection: a preliminary study. Anal. Bioanal. Chem. 407(12), 3271-3275 (2015).

31. Li S, Zhang Y, Xu J et al. Noninvasive prostate cancer screening based on serum surface-enhanced Raman spectroscopy and support vector machine. Appl. Phys. Lett. 105(9), 091104 (2014).

32. Leopold N, Lendl B. A new method for fast preparation of highly surface-enhanced raman scattering (SERS) active silver colloids at room temperature by reduction of silver nitrate with hydroxylamine hydrochloride. J. Phys. Chem. B 107(24), 5723-5727 (2003).

33. Westley C, Xu Y, Thilaganathan B, Carnell AJ, Turner NJ, Goodacre R. Absolute quantification of uric acid in human urine using surface enhanced raman scattering with the standard addition method. Anal. Chem. 89(4), 2472-2477 (2017).

34. Del Mistro G, Cervo S, Mansutti E et al. Surface-enhanced Raman spectroscopy of urine for prostate cancer detection: a preliminary study. Anal. Bioanal. Chem. 407(12), 3271-3275 (2015).

35. Howard SC, Jones DP, Pui C-H. The tumor lysis syndrome. N. Engl. J. Med. 364(19), 1844-1854 (2011).

36. Sato A, Shirota T, Shinoda T et al. Hyperuricemia in patients with hyperthyroidism due to Graves' disease. Metabolism 44(2), 207-211 (1995).

37. Wortmann RL. Gout and hyperuricemia. Curr. Opin. Rheumatol. 14(3), 281-286 (2002).

38. Feng S, Wang W, Tai IT, Chen G, Chen R, Zeng H. Label-free surface-enhanced Raman spectroscopy for detection of colorectal cancer and precursor lesions using blood plasma. Biomed. Opt. Express 6(9), 3494-3502 (2015).

39. Feng S, Huang S, Lin D et al. Surface-enhanced Raman spectroscopy of saliva proteins for the noninvasive differentiation of benign and malignant breast tumors. Int. J. Nanomedicine 10, 537-547 (2015).

40. Li X, Yang T, Li S et al. Noninvasive liver diseases detection based on serum surface enhanced Raman spectroscopy and statistical analysis. Opt. Express 23(14), 18361-18372 (2015).

41. Bonifacio A, Dalla Marta S, Spizzo R et al. Surface-enhanced Raman spectroscopy of blood plasma and serum using Ag and Au nanoparticles: a systematic study. Anal. Bioanal. Chem. 406(9-10), 2355-2365 (2014).

42. Li X, Yang T, Li S, Wang D, Guan D. Detecting esophageal cancer using surface-enhanced raman spectroscopy (SERS) of serum coupled with hierarchical cluster analysis and principal component analysis. Appl. Spectrosc. 69(11), 1334-1341 (2015). 
43. Shao X, Pan J, Wang Y et al. Evaluation of expressed prostatic secretion and serum using surface-enhanced Raman spectroscopy for the noninvasive detection of prostate cancer, a preliminary study. Nanomed. Nanotechnol. Biol. Med. 13(3), 1051-1059 (2017).

44. Holmström B, Johansson M, Bergh A, Stenman U-H, Hallmans G, Stattin P. prostate-specific antigen for early detection of prostate cancer: longitudinal study. BMJ 339, b3537 (2009).

45. Lokich E, Palisoul M, Romano $\mathrm{N}$ et al. Assessing the risk of ovarian malignancy algorithm for the conservative management of women with a pelvic mass. Gynecol. Oncol. 139(2), 248-252 (2015).

46. Molina R, Marrades RM, Auge JM et al. Assessment of a combined panel of six serum tumor markers for lung cancer. Am. J. Respir. Crit. Care Med. 193(4), 427-437 (2016).

47. Catalona WJ, Partin AW, Slawin KM et al. Use of the percentage of free prostate-specific antigen to enhance differentiation of prostate cancer from benign prostatic disease: a prospective multicenter clinical trial. JAMA 279(19), 1542-1547 (1998).

48. Gronberg H, Adolfsson J, Aly M et al. Prostate cancer screening in men aged 50-69 years (STHLM3): a prospective population-based diagnostic study. Lancet Oncol. 16(16), 1667-1676 (2015).

49. Strom P, Nordstrom T, Gronberg H, Eklund M. The Stockholm-3 model for prostate cancer detection: algorithm update, biomarker contribution, and reflex test potential. Eur. Urol. doi:10.1016/j.eururo.2017.12.028 (2018). (Epub ahead of print).

50. Holdenrieder S, Pagliaro L, Morgenstern D, Dayyani F. Clinically meaningful use of blood tumor markers in oncology. Biomed. Res. Int. 2016, 9795269 (2016). 
(

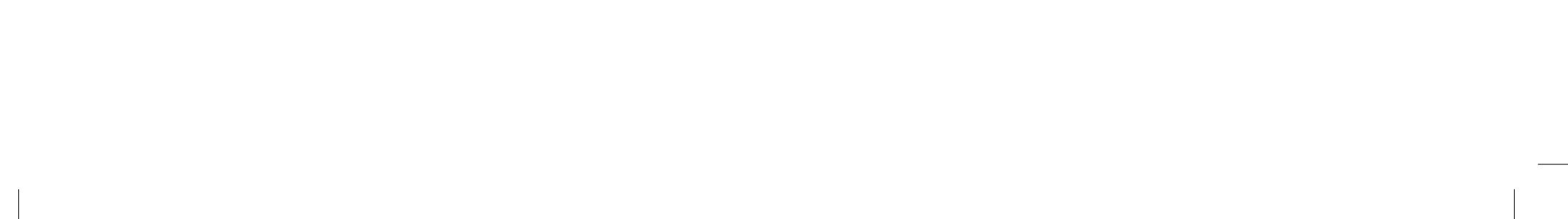

